\title{
Perfil Clínico-Funcional de idosas residentes em uma Instituição de Longa Permanência do município de Fortaleza
}

\author{
Clinical and Functional Profile of the elderly residents of a Long-Term Institution from Fortaleza \\ Perfil Clínico-Funcional de ancianas residentes en una Institución de Larga Estadía de Fortaleza
}

Recebido: 04/12/2021 | Revisado: 13/12/2021 | Aceito: 25/01/2022 | Publicado: 26/01/2022

\author{
Maria Beatriz Nunes de Carvalho \\ ORCID: https://orcid.org/0000-0003-1159-680X \\ Universidade Estadual do Ceará, Brasil \\ E-mail: bnuunes15@gmail.com \\ Geraldo Lucas Alves Monte \\ ORCID: https://orcid.org/0000-0002-9655-2656 \\ Universidade Estadual do Ceará, Brasil \\ E-mail: lucasmonte.a@gmail.com \\ Maria Célia Pinheiro da Cunha \\ ORCID: https://orcid.org/0000-0003-0490-5723 \\ Universidade Estadual do Ceará, Brasil \\ E-mail: mariaceliapinheirodc@gmail.com \\ Christian Raphael Fernandes Almeida \\ ORCID: https://orcid.org/0000-0002-3403-1791 \\ Universidade Estadual do Ceará, Brasil \\ E-mail: christian.raphael00@gmail.com \\ Marina Valente Mascarenhas \\ ORCID: https://orcid.org/0000-0001-7248-1649 \\ Universidade Estadual do Ceará, Brasil \\ E-mail: mvalentemascarenhas@gmail.com \\ Rhanna Emanuela Fontenele Lima de Carvalho \\ ORCID: https://orcid.org/0000-0002-3406-9685 \\ Universidade Estadual do Ceará, Brasil \\ E-mail: rhannalima@gmail.com
}

\begin{abstract}
Resumo
O objetivo deste estudo foi identificar o perfil clínico-funcional de idosas residentes de uma instituição de longa permanência por meio do instrumento Índice de Vulnerabilidade Clínico Funcional-20 (IVCF-20). Compuseram a amostra 29 idosas, com idade a partir de 65 anos. Tal instrumento é composto por 20 questões que contemplam aspectos multidimensionais da condição de saúde do idoso e o classifica em robusto, com risco de fragilização ou frágil. Os resultados obtidos no presente estudo categorizaram majoritariamente idosas com risco de fragilização.
\end{abstract}

Palavras-chave: Idoso; Instituição de longa permanência para idosos; Perfil de saúde.

\begin{abstract}
The objective of this study was to identify the clinical-functional profile of elderly residents of a long-term institution using the Functional Clinical Vulnerability Index-20 (IVCF-20). The sample was composed of 29 elderly women, aged 65 and over. The instrument used is composed of 20 questions that contemplate multidimensional aspects of the health condition of the elderly and classifies them as robust, with risk of fragility or fragility. The results obtained in this study categorized mostly elderly women at risk of frailty.

Keywords: Elderly; Homes for the aged; Health profile.

\section{Resumen}

El objetivo de este estudio fue identificar el perfil clínico-funcional de ancianas residentes de una institución de larga estadía utilizando el Î́ndice de Vulnerabilidad Clínica Funcional-20 (IVCF-20). La muestra estuvo compuesta por 29 mujeres mayores de 65 años y más. Este instrumento es compuesto por 20 preguntas que contemplan aspectos multidimensional del estado de salud de las personas mayores y las clasifica como robustas, con riesgo de fragilidad o fragilidad. Los resultados obtenidos en el presente estudio categorizaron mayoritariamente a mujeres ancianas con riesgo de fragilidad.
\end{abstract}

Palabras clave: Anciano; Hogares para ancianos; Perfil de salud. 


\section{Introdução}

Envelhecer é um fenômeno inerente a todos os seres humanos, que ocorre de maneira gradual e possui um impacto significativo nas sociedades do mundo inteiro. De acordo com o Estatuto do Idoso (2013), no Brasil há aproximadamente 20 milhões de pessoas com idade igual ou superior a 60 (sessenta) anos, e para 2025, esse número chegará a 32 milhões, passando a ocupar o $6^{\circ}$ lugar no mundo em número de idosos.

A chegada da senilidade está intimamente ligada aos processos de fragilização, devido às diversas alterações fisiológicas que podem causar grandes limitações nos idosos, comprometendo o bom funcionamento do seu corpo e ocasionando distúrbios locomotores, emocionais e cognitivos, afetando, assim, a sua autonomia e independência, tornando-o vulnerável e diminuindo a sua qualidade de vida.

No envelhecimento humano, estão envolvidos fatores sociais, ambientais, biológicos e psicológicos, além das mudanças culturais, econômicas e institucionais (Oliveira et al., 2019). Tal fenômeno tende a elevar o número de doenças crônicas e dependências funcionais nos idosos, contribuindo, assim, com o aumento da procura e utilização dos serviços de atenção à saúde.

À vista disso, é relevante analisar aspectos do processo de envelhecer, como a fragilidade, que é multidimensional e responsável pela redução da reserva homeostática e/ou capacidade de adaptação a alterações biopsicossociais, que aumentam a suscetibilidade ao declínio funcional (Moraes et al., 2016).

Assim, intensifica-se a busca por serviços especializados em saúde do idoso, devido à grande necessidade de cuidados individualizados e específicos para com os mesmos, como Centros de Convivência, Centros Dia e Instituições de Longa Permanência para Idosos (ILPI).

Diante de algumas circunstâncias e dificuldades com o processo do envelhecimento, os familiares recorrem às ILPIs, que são "instituições governamentais ou não governamentais, de caráter residencial, destinadas a domicílio coletivo de pessoas com idade igual ou superior a 60 anos, com ou sem suporte familiar, em condição de liberdade, dignidade e cidadania” (Brasil, 2005, p. 1).

A fim de qualificar o cuidado fornecido, as ILPIs devem possuir equipes multiprofissionais compostas por médico, nutricionista, psicólogo, assistente social, fisioterapeuta, educador físico, cuidadores, como também enfermeiro e técnicos de enfermagem (Salcher et al., 2015). Destacando-se o profissional de enfermagem que possui papel fundamental diante ao cuidado direto e contínuo à pessoa idosa.

Destarte, é fundamental que os profissionais de saúde atuantes nessas ILPIs passem a utilizar instrumentos de triagem rápida, capazes de reconhecer e avaliar o idoso de risco, a fim de proporcionar um cuidado melhor e mais eficaz, contribuindo, assim, para a garantia da sua qualidade de vida.

Dessa forma, torna-se importante a aplicabilidade desses instrumentos para o reconhecimento da capacidade física dos idosos, em especial, aqueles que se encontram institucionalizados nas ILPIs, pois os cuidados e a atenção principal podem ser redirecionados para aqueles que mais necessitam, além de poder promover autonomia para aqueles que estão em suas plenas capacidades físicas de execução de atividades (Paula et al., 2017).

Por conseguinte, faz-se necessário o uso do Índice de Vulnerabilidade Clínico-Funcional-20 (IVCF-20), instrumento de triagem interdisciplinar, de rápida e fácil aplicação, que avalia tanto a dimensão física quanto cognitiva e psicológica do idoso, isto é, contempla aspectos multidimensionais da sua condição de saúde (Ribeiro et al., 2020). Foi construído por equipe multidisciplinar especializada na atenção à pessoa idosa, com a contribuição de Agentes Comunitários de Saúde (ACS), além de auxiliares, técnicos de enfermagem e gestores (Moraes et al., 2016).

O IVCF-20 foi considerado válido e confiável, segundo os dados psicométricos obtidos na pesquisa de Ribeiro et al. (2020), o que indica que é um instrumento capaz de identificar a fragilidade do idoso na Atenção Primária à Saúde (APS). Logo, tornou-se o primeiro instrumento brasileiro de identificação simples e rápida do idoso frágil na Atenção Básica à Saúde, com 
caráter multidimensional, capaz de ser utilizado por qualquer profissional de saúde capacitado, e com a finalidade de identificar se há a necessidade de encaminhamento para a atenção secundária.

Portanto, o estudo teve como objetivo identificar o perfil clínico-funcional de idosas residentes de uma instituição de longa permanência por meio do instrumento IVCF-20.

\section{Metodologia}

Trata-se de estudo transversal de abordagem quantitativa, que se trata da coleta de dados em determinado período ou ponto temporal (Polit \& Beck, 2011), realizado com idosas residentes de uma ILPI filantrópica de Fortaleza-CE, como parte das atividades de extensão do Programa de Educação Tutorial (PET) do curso de Enfermagem da Universidade Estadual do Ceará. O local do estudo foi uma instituição que se mantinha a partir da colaboração financeira das idosas, o valor era calculado a partir da condição financeira da família, assim como, de doações de entidades ou pessoas físicas.

A coleta sucedeu-se nas segundas-feiras à tarde, horário destinado ao projeto de extensão do programa. $\mathrm{O}$ ambiente da ILPI onde ocorreu a coleta foi o espaço coletivo das idosas, local destinado às atividades em grupos, refeições e momentos dinâmicos. Dessa forma, os bolsistas dividiram-se em duas equipes para as atividades de extensão, compostas por seis membros, que se alternavam semanalmente para a aplicação do instrumento. O tempo de coleta com cada idosa foi de aproximadamente 30 minutos.

Os dados foram obtidos por meio do uso do questionário Índice de Vulnerabilidade Clínico Funcional-20 (IVCF-20) aplicado pelos bolsistas do programa, no mês de outubro de 2018. O IVCF-20 foi desenvolvido de maneira interdisciplinar, por profissionais especialistas em geriatria e gerontologia, assim como por gestores de serviços de Atenção Básica à Saúde e do Núcleo de Apoio à Saúde da Família - NASF, atendendo, assim, realidades diversas da multiprofissionalidade (Moraes \& Carmo et al. 2016). Posteriormente, validado por Moraes, Carmo et al. (2016), obtendo alto grau de validade e confiabilidade.

O questionário contempla aspectos multidimensionais da condição de saúde do idoso, sendo constituído por 20 questões distribuídas em sete domínios: autopercepção da saúde, atividades de vida diária, cognição, humor, mobilidade, comunicação e comorbidades múltiplas. Somando-se ao final de todas as seções o máximo de 40 pontos. A depender do valor final obtido o idoso pode ser classificado em idoso robusto - escores de 0 a 6 pontos -, em risco de fragilização - de 7 a 14 pontos - ou frágil quando o total de escores é $\geq 15$ pontos -; considera-se que quanto maior o valor, maior é o declínio de vulnerabilidade clínicofuncional deste idoso (Moraes \& Carmo et al. 2016).

A ILPI referente no estudo contava exclusivamente com o público feminino, dessa forma, 39 idosas residiam na instituição. Assim, os critérios de inclusão estabelecidos foram idosas no recorte etário a partir de 65 anos e que estivessem presentes no dia da coleta. Foram excluídas do quantitativo amostral as residentes que possuíam déficits cognitivos diagnosticados nos prontuários e/ou que não se encontravam orientadas para responder as perguntas no momento da aplicação do instrumento. Diante disso, 29 idosas compuseram a amostra, mediante assinatura do Termo de Consentimento Livre e Esclarecido (TCLE) pelos idosos e ou responsável legal.

Os resultados obtidos foram tabulados no programa Microsoft Excel 2018. A pesquisa seguiu os preceitos éticos e legais de estudos com seres humanos conforme a Resolução n 466/2012 do Conselho Nacional de Saúde, tendo aprovação no Comitê de Ética em Pesquisa da Universidade Estadual do Ceará com o parecer $n^{\circ} 3.480 .627$ e Certificado de Apresentação para Apreciação Ética (CAAE) no 17075019.0.0000.5534.

\section{Resultados}

Em relação aos resultados que caracterizam a população idosa residente da ILPI, $14(48,2 \%)$ encontravam-se na faixa etária de 75 a 84 anos, $10(34,4 \%)$ na faixa etária de $\geq 85$ anos e $5(17,2 \%)$ na faixa etária de 60 a 74 anos. 
Destas, observou-se que 12 (41,3\%) estavam classificadas em risco de fragilização, $7(24,1 \%)$ robustas e $10(34,4 \%)$ frágeis. No que diz respeito à idade, $6(60 \%)$ encontravam-se na faixa de $\geq 85$ anos para idosas frágeis; já para as classificadas em risco de fragilização, $6(50 \%)$ estavam na faixa de 75 a 84 anos; para as robustas, 4 (57,1\%) estavam na faixa etária de 75 a $84 \operatorname{anos}($ Tabela 1).

Tabela 1 - Distribuição da relação idade-classificação IVCF-20.

\begin{tabular}{c|c|c|c}
\hline Faixa Etária & Risco de fragilização & Robustas & Frágeis \\
\hline 60 a 74 anos & 2 & 3 & - \\
\hline 75 a 84 anos & 6 & 4 & 4 \\
\hline$\geq 85$ anos & 4 & - & 6 \\
\hline
\end{tabular}

Fonte: Autores (2021).

Quanto aos domínios do IVCF-20, em relação à autopercepção da saúde, 18 (62,1\%) consideraram seu estado clínico como 'Excelente, muito boa ou boa' (Tabela 2).

Tabela 2 - Domínio da autopercepção da saúde IVCF-20.

\begin{tabular}{c|c|c|}
\hline DOMÍNIO & ITEM & F(\%) \\
\hline \multirow{3}{*}{$\begin{array}{c}\text { AUTOPERCEPÇÃO DA } \\
\text { SAÚDE }\end{array}$} & $\begin{array}{c}\text { Em geral, comparando com outras pessoas de sua idade, você diria } \\
\text { que sua saúde é: }\end{array}$ & \\
\cline { 2 - 3 } & Excelente, muito boa ou boa & $18(62,1)$ \\
\cline { 2 - 3 } & Regular ou ruim & $11(37,9)$ \\
\hline
\end{tabular}

Legenda: F - frequência. Fonte: Autores (2021).

Ao analisar as Atividades de Vida Diária (AVD), constatou-se que 15 (51,7\%) não deixaram de realizar compras por causa de sua saúde ou condição física. Paralelo a esse questionamento, as outras se mostraram maiores, 18 (62,1\%) responderam que não deixaram de controlar seu dinheiro, gastos ou pagar as contas da sua casa devido a sua saúde ou condição física, 17 $(58,6 \%)$ disseram que não interromperam a realização de pequenos trabalhos domésticos e $21(72,4 \%)$ não deixaram de tomar banho sozinhas.

Em relação a cognição, 22 (75,8\%) relataram não haver fala de algum familiar ou amigo sobre estar ficando esquecida, $23(79,3 \%)$ afirmaram que o esquecimento está piorando nos últimos meses, assim como 25 (86,2\%) relataram o seu impedimento na realização de alguma atividade do cotidiano.

Para o humor, demonstrou-se que $25(86,2 \%)$ não sentiram desânimo, medo ou tristeza no último mês, contudo, 15 $(51,7 \%)$ responderam que perderam o interesse em realizar atividades anteriormente prazerosas.

No que diz respeito à mobilidade, observou-se que $18(62,1 \%)$ possuem capacidade de elevar os braços acima do ombro e $22(75,8 \%)$ conseguem manusear ou segurar pequenos objetos, porém, 15 (51,7\%) apresentam dificuldade para caminhar capaz de impedir alguma atividade do cotidiano. Ademais, 21 (72,4\%) idosas não tiveram duas ou mais quedas no último ano e relataram não perder urina ou fezes de forma involuntária.

Referente à comunicação, 21 (72,4\%) idosas não possuem problemas de visão ou audição capazes de impedi-las de realizar alguma atividade do cotidiano. 
Para as comorbidades múltiplas, constatou-se que $25(86,2 \%)$ idosas não dispõem de nenhuma das três condições avaliadas: cinco ou mais doenças crônicas; uso regular de cinco ou mais medicamentos diferentes, todo dia; e internação nos últimos 6 meses (Quadro 1).

Quadro 1 - Distribuição dos domínios do IVCF-20.

\begin{tabular}{|c|c|c|c|}
\hline DOMÍNIOS & ITENS & $\begin{array}{l}\mathrm{NÃO} \\
\mathrm{F}(\%)\end{array}$ & $\begin{array}{l}\mathrm{SIM} \\
\mathrm{F}(\%)\end{array}$ \\
\hline \multirow{4}{*}{$\begin{array}{l}\text { ATIVIDADES DE } \\
\text { VIDA DIÁRIA }\end{array}$} & Por causa de sua saúde ou condição física, você deixou de fazer compras? & $15(21,7)$ & $14(48,3)$ \\
\hline & $\begin{array}{l}\text { Por causa de sua saúde ou condição física, você deixou de controlar seu } \\
\text { dinheiro, gastos ou pagar as contas de sua casa? }\end{array}$ & $18(62,1)$ & $11(37,9)$ \\
\hline & $\begin{array}{l}\text { Por causa de sua saúde ou condição física, você deixou de realizar } \\
\text { pequenos trabalhos domésticos, como lavar louça, arrumar a casa ou fazer } \\
\text { limpeza leve? }\end{array}$ & $17(58,6)$ & $12(41,4)$ \\
\hline & $\begin{array}{l}\text { Por causa de sua saúde ou condição física, você deixou de tomar banho } \\
\text { sozinho? }\end{array}$ & $21(72,4)$ & $8(27,6)$ \\
\hline \multirow[t]{3}{*}{ COGNIÇÃO } & Algum familiar ou amigo falou que você está ficando esquecido? & $22(75,8)$ & $7(24,2)$ \\
\hline & Este esquecimento está piorando nos últimos meses? & $23(79,3)$ & $6(20,7)$ \\
\hline & $\begin{array}{l}\text { Este esquecimento está impedindo a realização de alguma atividade do } \\
\text { cotidiano? }\end{array}$ & $25(86,2)$ & $4(13,8)$ \\
\hline \multirow[t]{2}{*}{ HUMOR } & No último mês, você ficou com desânimo, tristeza ou desesperança? & $14(48,3)$ & $15(51,7)$ \\
\hline & $\begin{array}{l}\text { No último mês, você perdeu o interesse em atividades anteriormente } \\
\text { prazerosas? }\end{array}$ & $16(55,1)$ & $13(44,9)$ \\
\hline \multirow[t]{6}{*}{ MOBILIDADE } & Você é incapaz de elevar os braços acima do nível do ombro? & $18(62,1)$ & $11(37,9)$ \\
\hline & Você é incapaz de manusear ou segurar pequenos objetos? & $22(75,8)$ & $7(24,2)$ \\
\hline & $\begin{array}{l}\text { Você tem alguma das quatro condições (Perda de peso não intencional de } \\
4,5 \mathrm{~kg} \text { ou } 5 \% \text { do peso corporal no último ano ou } 6 \mathrm{~kg} \text { nos últimos } 6 \text { meses } \\
\text { ou } 3 \mathrm{~kg} \text { no último mês; IMC }<22 \mathrm{~kg} / \mathrm{m}^{2} \text {; Circunferência da panturrilha } \\
<31 \mathrm{~cm} \text {; Tempo gasto no teste de velocidade da marcha }(4 \mathrm{~m})>5 \text { segundos? }\end{array}$ & $27(93,1)$ & $2(6,9)$ \\
\hline & $\begin{array}{l}\text { Você tem dificuldade para caminhar capaz de impedir a realização de } \\
\text { alguma atividade do cotidiano? }\end{array}$ & $14(48,3)$ & $15(51,7)$ \\
\hline & Você teve duas ou mais quedas no último ano? & $22(75,8)$ & $7(24,2)$ \\
\hline & Você perde urina ou fezes, sem querer, em algum momento? & $22(75,8)$ & $7(24,2)$ \\
\hline \multirow[t]{2}{*}{ COMUNICAÇÃO } & $\begin{array}{l}\text { Você tem problemas de visão capazes de impedir a realização de alguma } \\
\text { atividade do cotidiano? }\end{array}$ & $21(72,4)$ & $8(27,6)$ \\
\hline & $\begin{array}{l}\text { Você tem problemas de audição capazes de impedir a realização de alguma } \\
\text { atividade do cotidiano? }\end{array}$ & $21(72,4)$ & $8(27,6)$ \\
\hline $\begin{array}{l}\text { COMORBIDADES } \\
\text { MÚLTIPLAS }\end{array}$ & $\begin{array}{l}\text { Você tem alguma das três condições (Cinco ou mais doenças crônicas; Uso } \\
\text { regular de cinco ou mais medicamentos diferentes, todo dia; Internação } \\
\text { recente, nos últimos } 6 \text { meses)? }\end{array}$ & $25(86,2)$ & $4(13,8)$ \\
\hline
\end{tabular}

Legenda: F - frequência. Fonte: Autores (2021).

\section{Discussão}

Apesar da ILPI estudada ser exclusivamente composta por mulheres acima de 75 anos, o público majoritário das ILPI brasileiras é também o feminino, aspecto que pode ser justificado pela maior longevidade desse grupo. Alicerçada a essa perspectiva, Camarano e Barbosa (2016) salientam que uma pesquisa realizada pelo Instituto de Pesquisa Econômica Aplicada (Ipea) demonstrou que, no Brasil, as mulheres totalizavam 57,6\% da população de idosos residentes em instituições de longa permanência.

Os dados predominantes relacionados ao público do estudo constataram que $14(48,2 \%)$ das idosas que residiam na ILPI pesquisada possuíam idades entre 75 e 84 anos e 12 (41,3\%) encontravam-se, a partir da aplicação do IVCF-20, 
classificadas em risco de fragilização. Dessa forma, um estudo desenvolvido por Moreira et al. (2016) corroborou um perfil etário semelhante, contudo constatou prevalência elevada de idosos frágeis e ausência de idosos robustos. Outras pesquisas realizadas em ILPIs mostraram resultados equivalentes, com quantitativos predominantes de idosos frágeis (Barbosa et al., 2020; Fernandes et al., 2020; Fluetti et al., 2018).

O predomínio da robustez em idosos é uma característica relacionada ao envelhecimento bem-sucedido. À vista disso, Maia et al. (2020) evidenciam a presença desses indivíduos na atenção primária à saúde, principalmente na faixa etária entre 60 e 74 anos devido à ausência de comorbidades e incapacidades associadas. Todavia, é comum que o perfil de capacidade funcional seja modificado com o passar da idade.

Quanto aos resultados por domínio da IVCF-20, a autopercepção da saúde é um importante indicador a ser avaliado no que tange ao impacto de doenças crônicas em idosos, além de conseguir mensurar seu bem-estar físico, emocional e social. No atual estudo houve prevalência de $62,1 \%$ no que retrata essa autopercepção como excelente, muito boa ou boa. Um estudo realizado em um centro de convivência comunitário no Piaú com 216 idosos teve resultados semelhantes, com prevalência do sexo feminino, e com a utilização do mesmo instrumento, percebeu-se que a autopercepção da saúde foi excelente, muito boa ou boa em 66,7\% da amostra, contabilizando cerca de 144 idosas com idade média de 70,5 anos (Oliveira et al., 2020).

Cabe ressaltar que a literatura aponta que idosos com melhor índice de autopercepção da saúde têm menores riscos de mortalidade (Ribeiro et al., 2018). No Rio Grande do Sul, o estudo realizado com 305 idosos apresentou resultados positivos quanto à autopercepção da saúde, onde cerca de 51,8\% a consideravam excelente, boa ou muito boa (Silva et al., 2020).

Segundo Lopes e Santos (2015), a capacidade de executar AVD é o mais importante indicador de funcionalidade em idosos. A sua incapacidade funcional é definida pela dificuldade do indivíduo ou necessidade de ajuda para executar tarefas básicas ou mais complexas no seu dia a dia, podendo predispor o idoso à fragilidade e dependência (Farías-Antúnez et al., 2018; Carneiro, Vilela, \& Meira, 2016).

Em um estudo realizado por Oliveira e Mattos (2012) com 154 idosos institucionalizados no município de Cuiabá-MT que utilizou as escalas de Katz e de Lawton, identificou que a prevalência de dependência para Atividades Básicas de Vida Diária (ABVD) foi de 44\% e em Atividades Instrumentais de Vida Diária (AIVD), correspondeu a 88,7\%, sendo que indivíduos do sexo feminino e que referiram saúde regular/ruim apresentaram maior probabilidade de dependência para AVD. Ademais, os autores concluíram que a prevalência de dependência para AVD em idosos institucionalizados foi 6,4 e 3,0 vezes maior respectivamente, do que quando observada em idosos da comunidade.

Nesse sentido, o resultado obtido no atual estudo, o qual predominou a independência para AVD, não vai de encontro com a maioria dos estudos evidenciados na literatura que aplicaram as escalas de Katz e de Lawton, pois seus resultados são caracterizados por idosos dependentes para AVD Básica e Instrumental (Melo et al., 2017; Oliveira \& Tavares, 2014; Santiago et al., 2016).

Apesar da condição de institucionalização dos idosos, eles possuem a expectativa de realizar atividades que lhes eram inerentes em sua idade produtiva. Contudo, a depender de como algumas instituições desenvolvem suas atividades diárias, o mesmo pode desenvolver um grau de dependência, visto que, os próprios funcionários preferem ajudar os idosos em suas atividades, quando esses já apresentam inabilidade para executar tarefas simples, embora não sejam incapazes para fazê-las (Soares et al., 2018; Carneiro et al., 2016).

Quanto a cognição no indivíduo idoso, a mesma torna-se um elemento importante a ser mensurado, visto que ocorre um declínio nas atividades mentais com o evoluir da idade, e pode ser evidenciada por meio de esquecimento, dificuldade em realizar atividades matemáticas e afins, além disso, pode estar associado ao uso de medicamentos e psicopatologias (Ribeiro et al., 2018; Oliveira et al., 2020). 
No atual estudo houve uma prevalência positiva significativa na cognição das idosas estudadas com percentual mínimo de 75,8\% em uma das perguntas que são realizadas nesse domínio do instrumento. Comparado a um estudo realizado por Silva et al. (2020) em domicílio com 304 idosos do Rio Grande do Sul, percebeu-se que 75,1\% não apresentavam nenhum declínio na cognição.

No entanto, vale ressaltar que pode existir uma diferença entre ambos resultados, tendo em vista que a realidade das ILPIs é diferente da dos domicílios, nesse caso, pode-se perceber que houve um encontro nas informações, mas, não assegura que esses números serão sempre semelhantes, fazendo com que tais resultados sejam designados à ILPI estudada e não uma realidade global.

Cabe salientar que as instituições de longa permanência muitas vezes favorecem a depreciação do humor em idosos, por estarem longe da família, de seus lares e convívio social. Estudos mostraram que o aparecimento de humor depressivo em idosos institucionalizados é maior do que comparado aqueles que vivem com seus familiares.

$\mathrm{Na}$ Bahia, em uma ILPI filantrópica Guimarães et al. (2019) evidenciou que o estado de humor negativo foi presente em 54,8\% dos 42 idosos avaliados. No entanto, no presente estudo foi possível perceber que o humor se manteve em um estado mediano, sendo positivo em mais da metade da população. No Piauí, no estudo realizado por Silva et al. (2020) em um centro de convivência comunitário para idosos também foi possível evidenciar esse humor positivo em $74,1 \%$ da amostra composta por 216 idosos.

Pode-se perceber que há uma diferença nos resultados quando comparados com outros meios de estudo, havendo assim, a necessidade de entender e adequar-se à realidade de cada instituição, podendo ser a do estudo atual, uma exceção das que estão espalhadas pelo país. Salienta-se que o tipo de instituição também pode favorecer esse tipo de comportamento, no caso do estudo por tratar-se de uma instituição onde havia contribuição de alguma forma da família, a mesma possui um contato com as tais, e, portanto, podem ter um elo de ligação maior, contribuindo para os resultados positivos do humor nas idosas avaliadas.

A mobilidade é um fator importante na avaliação da qualidade de vida e dependência do idoso. Para avaliá-la pode ser usado também um instrumento denominado "Timed Up and Go" (TUG) que segundo Carneiro e Meira (2016) analisa a mobilidade funcional de idosos levando em conta equilíbrio, marcha e capacidade funcional.

Em um estudo realizado por Fernandes et al. (2020), utilizando o TUG foi identificado que a maioria dos idosos possuíam velocidade da marcha diminuída (a partir de 10 segundos), dos quais todos os idosos foram classificados como frágeis, além disso destes $85,7 \%$ dos idosos não relataram histórico de queda. O presente estudo diverge deste resultado, pois $93,1 \%$ das idosas não relataram marcha diminuída (> 5 segundos) e apenas 34,4\% foram classificadas como frágeis.

Concomitante a isto, é possível constatar que as idosas do presente estudo não apresentaram grande dificuldade no domínio de mobilidade relacionado a alcance e preensão, visto que 75,8\% conseguem manusear pequenos objetos e 48,3\% não possui dificuldade para caminhar, confirmando assim uma maior independência para atividades básicas e de vida diária.

Tal resultado corrobora com um estudo realizado na Bahia, que ao comparar idosos institucionalizados e não institucionalizados identificou que $88,2 \%$ dos idosos institucionalizados eram considerados independentes, em contraponto a 70,5\% dos não institucionalizados (Carneiro et al., 2016).

No que se refere às alterações fisiológicas próprias do envelhecimento, observa-se que estas podem comprometer as principais dimensões de desempenho dos idosos, entre elas a comunicação. À vista disso, Liljas et al. (2015) afirmam que a deterioração da função visual e auditiva contribui com o mau funcionamento físico, dificuldade de equilíbrio e marcha, ocorrência de quedas, incapacidades, baixo envolvimento social e uma vida mais inativa.

Em 2019, um estudo realizado com a análise das características da população idosa residente em Pombal-PB, constatou que $47,7 \%$ dos idosos possuía um maior comprometimento visual no olho direito e $43,3 \%$ no esquerdo e que tais achados foram 
identificados nos idosos em risco de fragilização, havendo associação entre o IVCF-20 e o Teste de Sinais e Snellen (Freitas \& Soares, 2019).

Na mesma abordagem, a distribuição dos idosos com relação ao Teste do Sussurro apontou predomínio de disfunção no ouvido direito em 40,5\% e 36\% no esquerdo na população de idosos em risco de fragilização, também com associação entre o IVCF-20 e o Teste do Sussurro para o ouvido direito e esquerdo (Freitas \& Soares, 2019). Os resultados achados na literatura divergem do estudo atual, visto que $72,4 \%$ das idosas não possuem deficiências visuais ou auditivas capazes de impedir a realização de suas atividades diárias.

O comprometimento do processo de comunicação implica na troca ineficaz de informações, desejos, ideias ou sentimentos vivenciados, como afirmam Moraes, Moraes, Santos et al. (2016), motivo que exige uma maior capacitação profissional para atuar nas diversas situações diárias utilizando estratégias de comunicação terapêutica, como escuta ativa, diálogo e apoio, que proporcionam um cuidado eficaz e, assim, a criação de vínculo com o idoso.

Quanto às comorbidades múltiplas, percebe-se que elas são consideradas prejudiciais para a saúde e estabilidade da qualidade de vida nos idosos, gerando, assim, uma diminuição da sua capacidade funcional.

À vista disso, um estudo realizado em 2019 com idosos residentes em instituições de longa permanência no município de Vitória da Conquista-BA, constatou que as patologias de maior frequência foram a hipertensão arterial e hipertensão arterial associada à acidente vascular encefálico, ambas correspondendo a 19,4\% de prevalência. Já a hipertensão arterial associada ao diabetes mellitus predominou em 16,1\% e a artrite em 14,5\%. Além disso, os autores salientaram, também, quanto à presença de tratamentos e os mais citados foram os medicamentosos associados à fisioterapia $(41,9 \%)$ e apenas medicamentosos, equivalente a 32,3\% (Oliveira, Araújo \& Reis, 2020).

Tais estudos diferem dos resultados obtidos na presente pesquisa, visto que $86,2 \%$ das idosas entrevistadas não apresentaram nenhuma das três condições avaliadas, possuindo, portanto, um nível de fragilidade menor. Apesar disso, o processo de envelhecimento, somado às alterações fisiológicas que acompanham os idosos, podem causar um declínio de sua saúde, provocando uma maior demanda de cuidado prestado nas instituições de longa permanência.

Nesse sentido, segundo Silva e Gutierrez (2018), as ILPIs devem atender às necessidades desses idosos, considerando suas histórias de vida, preservando sua autonomia, facilitando sua compreensão sobre o processo de envelhecimento e tornandoos protagonistas do seu processo de cuidar.

No entanto, observou-se como limitação do estudo o quantitativo amostral reduzido, que associado a divergência dos achados com a maioria das pesquisas atuais, trouxe a reflexão de que o estudo se trata de uma realidade específica de uma ILPI filantrópica do município de Fortaleza. Ademais, percebeu-se que seria pertinente a consulta ao prontuário das idosas, com a finalidade de melhor fundamentar os dados encontrados a partir da aplicação do instrumento.

\section{Considerações Finais}

O IVCF-20 mostrou-se um instrumento útil para a classificação do perfil clínico-funcional das idosas institucionalizadas, uma vez que possibilita traçar melhores estratégias de cuidado pautadas na singularidade das idosas, podendo, assim, ser utilizado amplamente tanto na Atenção Básica em Saúde, quanto em ILPIs.

Os resultados obtidos no presente estudo categorizaram majoritariamente idosas com perfil de risco de fragilização, assim como independentes para as atividades básicas e instrumentais de vida diária. Dentre os domínios explorados, apenas humor e cognição corroboraram com a literatura científica. Além disso, os resultados não podem ser generalizados, por se tratarem de uma condição singular de uma ILPI filantrópica do município de Fortaleza.

Dessa forma, diante do fenômeno de envelhecimento populacional presente no Brasil, constatou-se a importância do desenvolvimento de mais estudos e pesquisas na área, com objetivo de estimular profissionais de enfermagem a implementar em 
sua prática assistencial o uso de instrumentos que possam auxiliar o manejo da atenção em saúde a esse público, a fim de promover um cuidado integral e qualificado à população idosa.

\section{Agradecimentos}

Agradecemos a contribuição de cada bolsista, inclusive egressos, do PET Enfermagem da UECE que se debruçaram arduamente no desenvolvimento do projeto de extensão intitulado: Educação em Saúde em Instituição de Longa Permanência em Idosos: um olhar ao idoso institucionalizado. O empenho de todos os envolvidos no processo, desde a escolha do tipo de estudo, etapa da coleta dos dados, e por fim escrita deste artigo culminaram no avanço satisfatório dessa pesquisa. Ademais, o mais sincero agradecimento à nossa querida tutora Rhanna Emanuela que nos norteou durante esta trajetória.

\section{Referências}

Barbosa, L. M, Noronha, K., Camargos, M. C. S., \& Machado, C. J. (2020). Perfis de integração social entre idosos institucionalizados não frágeis no município de Natal, Rio Grande do Norte, Brasil. Ciência \& Saúde Coletiva, 25(6), 2017-2030. https://scielosp.org/pdf/csc/2020.v25n6/2017-2030/pt.

Brasil, Ministério da Saúde (2005). Agência Nacional de Vigilância Sanitária. RDC nº 283 de 26 de setembro de 2005. Diário Oficial da União. http://bvsms.saude.gov.br/bvs/saudelegis/anvisa/2005/res0283_26_09_2005.html

Camarano, A. A., \& Barbosa, P. (2016) Instituições de longa permanência para idosos no Brasil: o que se está falando? In: Alcântara, A.O., Camarano, A.A., \& Giacomin, K.C. (Orgs). Política nacional do idoso: velhas e novas questões, cap. 20 , 479-514. Ipea. https://www.ipea.gov.br/portal/images/stories/PDFs/livros/livros/161006_livro_politica_nacional_idosos.PDF.

Carneiro, D. N., Vilela, A. B., \& Meira, S. S. (2016). Avaliação do déficit cognitivo, mobilidade e atividades da vida diária entre idosos. Revista de APS Atenção Primária à Saúde, 19(2), 203-209. https://periodicos.ufjf.br/index.php/aps/article/view/15453.

Farías-Antúnez, S., Lima, N. P., Bierhals, I. O., Gomes, A. P., Vieira, L. S., \& Tomasi, E. (2018). Incapacidade funcional para atividades básicas e instrumentais da vida diária: um estudo de base populacional com idosos de Pelotas, Rio Grande do Sul, 2014. Epidemiologia e Serviços de Saúde, 27(2), e2017290. https://www.scielo.br/pdf/ress/v27n2/2237-9622-ress-27-02-e2017290.pdf.

Fernandes, L. C., Moura, L. R., Mesquita, G. L., Mesquita A. L., \& Baldoino, G. R. (2020) Avaliação da fragilidade e da mobilidade funcional de idosos institucionalizados na região Centro-Oeste do Brasil: um estudo transversal. Movimenta, 13(2), 192-203. https://www.revista.ueg.br/index.php/movimenta/article/view/9331/7703

Fluetti, M. T., Fhon, J. R. S., Oliveira, A. P., Chiquito, L. M. O., \& Margueet, S. (2018). Síndrome da fragilidade em idosos institucionalizados. Revista Brasileira de Geriatria e Gerontologia, 21(1), 62-71. 1809-9823-rbgg-21-01-00060.pdf (scielo.br).

Freitas, F. F. Q., \& Soares, S. M. (2019). Índice de vulnerabilidade clínico-funcional e as dimensões da funcionalidade em idosos. Revista da Rede de Enfermagem do Nordeste, 20(1), 1-9. http://www.repositorio.ufc.br/bitstream/riufc/41810/1/2019_art_ffqfreitas.pdf

Guimarães, L. A., Brito, T. A., Pithon, K. R, Jesus, C. L., Souto, C. L., Souza, S. J. N. et al. (2019). Sintomas depressivos e fatores associados em idosos residentes em instituição de longa permanência. Ciência \& Saúde Coletiva. 24(9), 3275-3282. https://www.scielo.br/pdf/csc/v24n9/1413-8123-csc-24-093275.pdf

Liljas, A. E. M., Wannamethee, S. G., Whincup, P. H., Papacosta, O., Walters, K., Iliffe, S., Lennon, L. T., Carvalho, L. A., \& Ramsay, S. E. (2015). Sociodemographic characteristics, lifestyle factors and burden of morbidity associated with self-reported hearing and vision impairments in older British community-dwelling men: a cross-sectional study. Journal of Public Health, 38(2), 21-28. https://pubmed.ncbi.nlm.nih.gov/26177816/

Lopes, G. L., \& Santos M. I. (2015). Funcionalidade de idosos cadastrados em uma unidade da Estratégia Saúde da Família segundo categorias da Classificação Internacional de Funcionalidade. Revista Brasileira de Geriatria e Gerontologia., 18(1), 71-83. https://www.scielo.br/pdf/rbgg/v18n1/1809-9823-rbgg-18-0100071.pdf

Maia, L. C., Colares, T. F. B., Moraes, E. N., Costa, S. M., \& Caldeira, A. P. (2020). Idosos robustos na atenção primária: fatores associados ao envelhecimento bem-sucedido. Revista de Saúde Pública, 54(35), 1-11. https://scielosp.org/pdf/rsp/2020.v54/35/pt.

Melo, B. R., Diniz, M. A., Casemiro, F. G., Figueiredo, L. C., Santos-Orlandi, A. A., Haas, V. J., Orlandi, F. S., \& Gratão, A. C. M. (2017). Avaliação cognitiva e funcional de idosos usuários do serviço público de saúde. Escola Anna Nery, 21(4), e20160388. https://www.scielo.br/pdf/ean/v21n4/pt_1414-8145-ean-21779465-EAN-2016-0388.pdf 
Moraes, E. N., Carmo, J. A., Moraes, F. L., Azevedo, R. S., Machado, C. J., \& Montilla, D. E. R. (2016). Índice de Vulnerabilidade Clínico Funcional-20 (IVCF-20): reconhecimento rápido do idoso frágil. Revista de Saúde Publica, 50(81), 1-10. https://www.scielo.br/pdf/rsp/v50/pt_0034-8910-rsp-S151887872016050006963.pdf

Moraes, E. N., Moraes, F. L., Santos, R. R., Bicalho, M. A. C., Machado, C. J., \& Romero D. E. (2016). A new proposal for the clinical-functional categorization of the elderly: Visual scale of frailty (VS-Frailty). The Journal of Aging Research \& Lifestyle, 5(1), 24-30. https://www.jarlife.net/1808-a-new-proposal-for-theclinical-functional-categorization-of-the-elderly-visual-scale-of-frailty-vs-frailty-html

Moreira, J. C., Albuquerque, E. R., Marques, C. B., Côrtes, M. C. J. W., \& Gontijo, E. D. (2016) Funcionalidade de idosos residentes em instituição de longa permanência e risco de quedas. Revista Médica de Minas Gerais, 2(8), 191-194. Recuperado em 10 março, 2021, de: RMMG - Revista Médica de Minas Gerais - Funcionalidade de idosos residentes em instituição de longa permanência e risco de quedas.

Oliveira, C. C. F., Araújo, J. B. O., \& Reis, L. A. (2020). Relação entre força muscular respiratória e funcionalidade em idosos residentes em instituições de longa permanência. Revista Saúde (Santa Maria), 46(2), 1-11. https://periodicos.ufsm.br/revistasaude/article/view/42460/pdf

Oliveira, C. E., Felipe, S. G., Silva, C. R., Carvalho, D. B., Silva-Júnior, F., Figueiredo, M. L., et al. (2020). Vulnerabilidade clínico-funcional de idosos em um centro de convivência. Acta Paulista de Enfermagem, 33(1), 1-8. https://www.scielo.br/pdf/ape/v33/1982-0194-ape-33-eAPE20190172.pdf

Oliveira, I. C., Vasconcelos, R. O., Beck, C. L. C., Silva, R. M., Coelho, A. P. F., \& Barros, I. F. O. (2019). Educação com equipe de enfermagem de instituição de longa permanência para idosos: relato de experiência. Advances in Nursing and Health, 2(1), 127-141. http://www.uel.br/revistas/uel/index.php/anh/article/view/38843/26665

Oliveira, P.B., \& Tavares, D.M. (2014). Condições de saúde de idosos residentes em Instituição de Longa Permanência segundo necessidades humanas básicas. Revista Brasileira de Enfermagem, 67(2), 241-246. https://www.scielo.br/pdf/reben/v67n2/0034-7167-reben-67-02-0241.pdf.

Oliveira, P.H., \& Mattos I.E. (2012). Prevalência e fatores associados à incapacidade funcional em idosos institucionalizados no Município de Cuiabá, Estado de Mato Grosso, Brasil, 2009-2010. Epidemiologia e Serviços de Saúde, 21(3), 395-406. http://scielo.iec.gov.br/pdf/ess/v21n3/v21n3a05.pdf

Paula, R. T., Sousa, M. E. F. P., Reis, T. M., Santos L. A. C., Resende, M. A., \& Souza, G. (2017). A atuação do enfermeiro diante a depressão em idosos institucionalizados: subsídios de prevenção. Revista Eletrônica Acervo Saúde. 11(1), 1053-1060. https://www.researchgate.net/publication/323853966_A_atuacao_do_enfermeiro_diante_a_depressao_em_idosos_institucionalizados_subsidios_de_prevencao

Polit, D.F., \& Beck, C.T. (2011). Fundamentos de pesquisa em enfermagem: avaliação de evidências para a prática clínica. Artmed.

Ribeiro, E. G., Matozinhos, F. P., Guimaraes, G. L., Couto A. M., Azevedo, R. S., \& Mendoza I. Y. Q. (2018). Self-perceived health and clinical functional vulnerability of the elderly in Belo Horizonte/Minas Gerais. Revista Brasileira de Enfermagem 71(2), 860-867. de: https://www.scielo.br/pdf/reben/v71s2/pt_0034-7167-reben-71-s2-0860.pdf

Ribeiro, E. G., Mendoza, I. Y. Q., Moraes, E. N., Alvarenga, M. R. M., Cintra, M. T. G., \& Guimarães G. L. (2020). Propriedades psicométricas do índice de vulnerabilidade clínico-funcional - 20 na Atenção Primária à Saúde. Revista Mineira de Enfermagem, 24(1), 1-8. https://cdn.publisher.gn1.link/reme.org.br/pdf/e1332.pdf

Salcher, E. B. G., Portella, M. R., \& Scortegagna, H. M. (2015). Cenários de instituições de longa permanência para idosos: retratos da realidade vivenciada por equipe multiprofissional. Revista Brasileira Geriatria Gerontologia, 18(2), 259-272. https://www.scielo.br/pdf/rbgg/v18n2/1809-9823-rbgg-18-02-00259.pdf

Santiago, L. M., Luz, L. L., Silva, J. F., Oliveira, P. H., Carmo, C. N., \& Mattos, I. E. (2016). Condições sociodemográficas e de saúde de idosos institucionalizados em municípios das regiões Sudeste e Centro-Oeste do Brasil. Geriatrics, Gerontology and. Aging, 10(2), 86-92. https://www.researchgate.net/publication/307916940_Condicoes_sociodemograficas_e_de_saude_de_idosos_institucionalizados_em_cidades_do_Sudeste_e _ Centro-Oeste_do_Brasil

Secretaria Executiva, Ministério da Saúde. (2013). Estatuto do Idoso. (3a ed.). Ministério da Saúde. https://bvsms.saude.gov.br/bvs/publicacoes/estatuto_idoso_3edicao.pdf

Silva, H. S., \& Gutierrez, B. A. O. (2018). A Educação como instrumento de mudança na prestação de cuidados para idosos. Educar em Revista, 34(67), 283296. https://www.scielo.br/pdf/er/v34n67/0104-4060-er-34-67-283.pdf

Silva, J. N., Leite, M. T., Gaviraghi, L. C., Kirsten, V. R., Kinalski, S. S., Hildebrandt, L. M., et al. (2020). Predicting dimensions of clinical functional conditions and cognition in the elderly. Revista Brasileira de Enfermagem, 73(3), 1-8. https://www.scielo.br/pdf/reben/v73s3/pt_0034-7167-reben-73-s3-e20190162.pdf

Soares, N. V., Corrêa, B. R. S., Fontana, R. T., Brum, Z. P., Guimarães, C. A., Silva, A. F., \& Rodrigues, F. C. P. (2018). Sentimentos, expectativas e adaptações de idosos internados em instituições de longa permanência. REME - Revista Mineira de Enfermagem, $22: \mathrm{e}-1124$. https://cdn.publisher.gn1.link/reme.org.br/pdf/en_e1124.pdf 